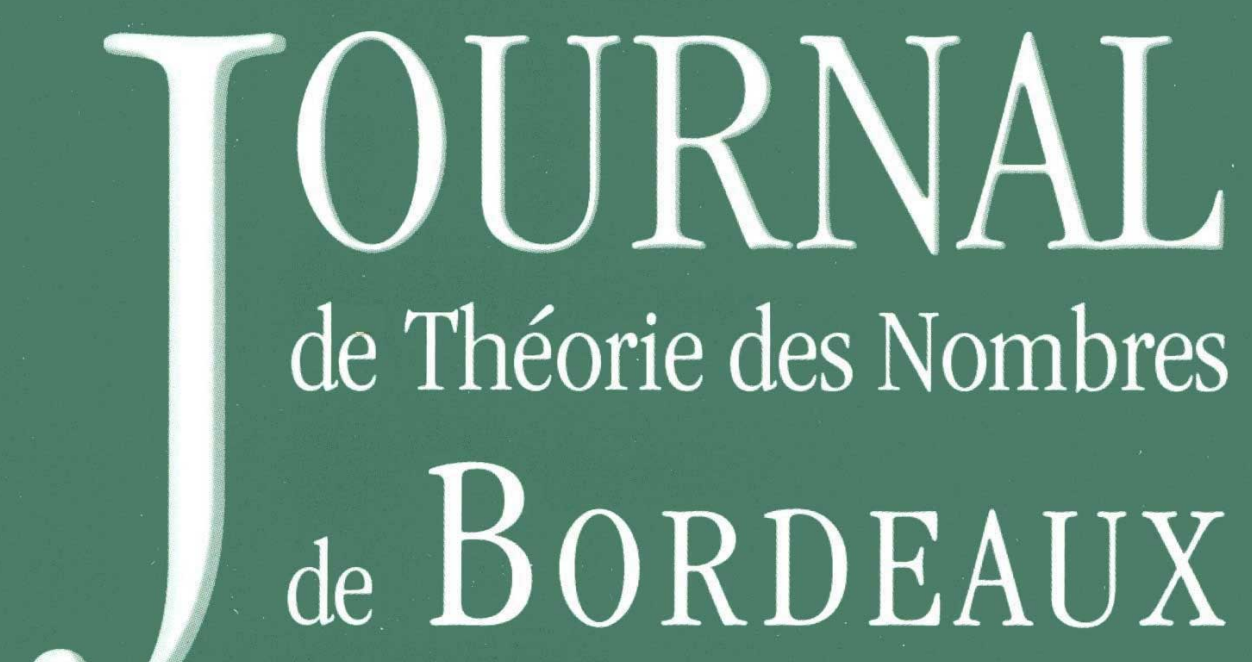

anciennement Séminaire de Théorie des Nombres de Bordeaux

Cornelis KRAAIKAMP, Thomas A. SCHMIDT et Ionica SMEETS

\title{
Tong's spectrum for Rosen continued fractions
}

Tome 19, no 3 (2007), p. 641-661.

<http://jtnb.cedram.org/item?id=JTNB_2007__19_3_641_0>

(C) Université Bordeaux 1, 2007, tous droits réservés.

L'accès aux articles de la revue «Journal de Théorie des Nombres de Bordeaux » (http://jtnb.cedram.org/), implique l'accord avec les conditions générales d'utilisation (http://jtnb.cedram. org/legal/). Toute reproduction en tout ou partie cet article sous quelque forme que ce soit pour tout usage autre que l'utilisation à fin strictement personnelle du copiste est constitutive d'une infraction pénale. Toute copie ou impression de ce fichier doit contenir la présente mention de copyright.

\section{cedram}




\title{
Tong's spectrum for Rosen continued fractions
}

\author{
par CoRnelis KRAAIKAMP, Thomas A. SCHMIDT et IONICA \\ SMEETS
}

\begin{abstract}
RÉsumÉ. Dans les années 90, J.C. Tong a donné une borne supérieure optimale pour le minimum de $k$ coefficients d'approximation consécutifs dans le cas des fractions continues à l'entier le plus proche. Nous généralisons ce type de résultat aux fractions continues de Rosen. Celles-ci constituent une famille infinie d'algorithmes de développement en fractions continues, où les quotients partiels sont certains entiers algébriques réels. Pour chacun de ces algorithmes nous déterminons la borne supérieure optimale de la valeur minimale des coefficients d'approximation pris en nombres consécutifs appropriés. Nous donnons aussi des résultats métriques pour des plages de "mauvaises" approximations successives de grande longueur.
\end{abstract}

ABSTRACT. In the 1990s, J.C. Tong gave a sharp upper bound on the minimum of $k$ consecutive approximation constants for the nearest integer continued fractions. We generalize this to the case of approximation by Rosen continued fraction expansions. The Rosen fractions are an infinite set of continued fraction algorithms, each giving expansions of real numbers in terms of certain algebraic integers. For each, we give a best possible upper bound for the minimum in appropriate consecutive blocks of approximation coefficients. We also obtain metrical results for large blocks of "bad" approximations.

\section{Introduction}

It is well-known that every $x \in[0,1) \backslash \mathbb{Q}$ has a unique (regular) continued fraction expansion of the form

$$
x=\frac{1}{a_{1}+\frac{1}{a_{2}+\ldots+\frac{1}{a_{n}+\ldots}}}=\left[a_{1}, a_{2}, \ldots, a_{n}, \ldots\right] .
$$

Manuscrit reçu le $1^{\mathrm{er}}$ septembre 2006.

The second author was supported by NWO Bezoekersbeurs B 61-505. 
Here the partial quotients $a_{n}$ are positive integers for $n \geq 1$. Finite truncation in (1.1) yields the convergents $p_{n} / q_{n}$ of $x$, i.e., for $n \geq 1$

$$
\frac{p_{n}}{q_{n}}=\frac{1}{a_{1}+\frac{1}{a_{2}+\ldots+\frac{1}{a_{n}}}}=\left[a_{1}, a_{2}, \ldots, a_{n}\right],
$$

and throughout it is assumed that $p_{n} / q_{n}$ is in its lowest terms. Note that (1.1) is a shorthand for $\lim _{n \rightarrow \infty} p_{n} / q_{n}=x$.

Underlying the regular continued fraction (RCF) expansion (1.1) is the $\operatorname{map} T:[0,1) \rightarrow[0,1)$, defined by

$$
T(x)=\frac{1}{x} \bmod 1=\frac{1}{x}-\left\lfloor\frac{1}{x}\right\rfloor, x \neq 0 ; \quad T(0)=0 .
$$

Here $\left\lfloor\frac{1}{x}\right\rfloor$ denotes the integer part of $\frac{1}{x}$. The RCF-convergents of $x \in[0,1) \backslash$ $\mathbb{Q}$ have strong approximation properties. We mention here that

$$
\left|x-\frac{p_{n}}{q_{n}}\right|<\frac{1}{q_{n}^{2}}, \text { for } n \geq 0,
$$

which implies, together with the well-known recurrence relations for the $p_{n}$ and $q_{n}$, that the rate of convergence of $p_{n} / q_{n}$ to $x$ is exponential (see e.g. $[\mathrm{DK}])$. One thus defines the approximation coefficients $\theta_{n}(x)$ of $x$ by $\theta_{n}=\theta_{n}(x)=q_{n}^{2}\left|x-p_{n} / q_{n}\right|, n \geq 0$. We usually suppress the dependence on $x$ in our notation.

For the RCF-expansion we have the following classical theorems by Borel (1905) and Hurwitz (1891) about the quality of the approximations.

Theorem 1. (Borel) For every irrational number $x$, and every $n \geq 1$

$$
\min \left\{\theta_{n-1}, \theta_{n}, \theta_{n+1}\right\}<\frac{1}{\sqrt{5}} .
$$

The constant $1 / \sqrt{5}$ is best possible.

Borel's result, together with a yet earlier result by Legendre $[\mathrm{L}]$, which states that if $p, q \in \mathbb{Z}, q>0$, and $\operatorname{gcd}(p, q)=1$, then

$$
\left|x-\frac{p}{q}\right|<\frac{1}{2 q^{2}} \quad \text { implies that } \quad\left(\begin{array}{c}
p \\
q
\end{array}\right)=\left(\begin{array}{c}
p_{n} \\
q_{n}
\end{array}\right), \quad \text { for some } n \geq 0,
$$

implies the following result by Hurwitz.

Theorem 2. (Hurwitz) For every irrational number $x$ there exist infinitely many pairs of integers $p$ and $q$, such that

$$
\left|x-\frac{p}{q}\right|<\frac{1}{\sqrt{5}} \frac{1}{q^{2}} .
$$

The constant $1 / \sqrt{5}$ is best possible. 
By removing all irrational numbers which are equivalent to the 'golden mean' $g=\frac{1}{2}(\sqrt{5}-1)$ (i.e., those irrationals whose RCF-expansion consists of $1 \mathrm{~s}$ from some moment on), we have that

$$
\left|x-\frac{p}{q}\right|<\frac{1}{\sqrt{8}} \frac{1}{q^{2}},
$$

for infinitely many pairs of integers $p$ and $q$. These constants $1 / \sqrt{5}$ and $1 / \sqrt{8}$ are the first two points in the so-called Markoff spectrum; see [CF] or [B] for further information on this spectrum, and the related Lagrange spectrum.

Note that the theorem of Borel does not suffice to prove Hurwitz's theorem; one needs Legendre's result to rule out the existence of rationals $p / q$ which are not RCF-convergents, but still satisfy $|x-p / q|<1 /\left(\sqrt{5} q^{2}\right)$.

In [T1, T2], Tong generalized Borel's results to the nearest integer continued fraction expansion (NICF). These are continued fractions of the form

$$
x=\frac{\varepsilon_{1}}{b_{1}+\frac{\varepsilon_{2}}{b_{2}+\ldots+\frac{\varepsilon_{n}}{b_{n}+\ldots}}},
$$

generated by the operator $T_{\frac{1}{2}}:\left[-\frac{1}{2}, \frac{1}{2}\right) \rightarrow\left[-\frac{1}{2}, \frac{1}{2}\right)$, defined by

$$
T_{\frac{1}{2}}(x)=\frac{\varepsilon}{x}-\left\lfloor\frac{\varepsilon}{x}+\frac{1}{2}\right\rfloor, x \neq 0 ; \quad T(0)=0,
$$

where $\varepsilon$ denotes the sign of $x$. Since the NICF-expansion of any number $x$ can be obtained from the RCF-expansion via a process called singularization (see $[\mathrm{DK}]$ or $[\mathrm{IK}]$ for details), the sequence of NICF-convergents $\left(r_{k} / s_{k}\right)_{k \geq 0}$ is a subsequence $\left(p_{n} / q_{n}\right)_{n \geq 0}$ of the sequence of RCF-convergents of $x$. Due to this, the approximation by NICF-convergents is faster; see e.g. $[\mathrm{A}]$, or $[\mathrm{IK}]$. In $[\mathrm{BJW}]$ it was shown that the approximation by NICF-convergents is also closer; for almost all $x$ one has that

$$
\lim _{k \rightarrow \infty} \frac{1}{k} \sum_{i=0}^{k-1} \vartheta_{k}=\frac{\sqrt{5}-2}{2 \log G}=0.24528 \ldots
$$

whereas

$$
\lim _{n \rightarrow \infty} \frac{1}{n} \sum_{i=0}^{k-1} \theta_{i}=\frac{1}{4 \log 2}=0.36067 \ldots,
$$

where $\vartheta_{k}=\vartheta_{k}(x)=s_{k}^{2}\left|x-r_{k} / s_{k}\right|$ is the $k$ th NICF-approximation coefficient of $x$, and $G=g+1$.

In contrast to this, it was shown in [JK] that for almost every $x$ there are infinitely many arbitrary large blocks of NICF-approximation coefficients $\vartheta_{n-1}, \ldots, \vartheta_{n+k}$, which are all larger than $1 / \sqrt{5}$. In spite of this, it is also 
shown in $[\mathrm{JK}]$ that for all irrational numbers $x$ there exist infinitely many $k$ for which $\vartheta_{k}<1 / \sqrt{5}$.

In [T1] and [T2], Tong sharpened the results from [JK], by showing that for the NICF there exists a 'pre-spectrum,' i.e., there exists a sequence of constants $\left(c_{k}\right)_{k \geq 1}$, monotonically decreasing to $1 / \sqrt{5}$, such that for all irrational numbers $x$ the minimum of any block of $k+2$ consecutive NICFapproximation coefficients is smaller than $c_{k}$.

Theorem 3. (Tong) For every irrational number $x$ and all positive integers $n$ and $k$ one has

$$
\min \left\{\vartheta_{n-1}, \vartheta_{n}, \ldots, \vartheta_{n+k}\right\}<\frac{1}{\sqrt{5}}+\frac{1}{\sqrt{5}}\left(\frac{3-\sqrt{5}}{2}\right)^{2 k+3} .
$$

The constant $c_{k}=\frac{1}{\sqrt{5}}+\frac{1}{\sqrt{5}}\left(\frac{3-\sqrt{5}}{2}\right)^{2 k+3}$ is best possible.

In [HK], Hartono and Kraaikamp showed how Tong's result follows from a geometrical approach based on the natural extension of the NICF. We further this approach to find Tong's spectrum for an infinite family of continued fractions generalizing the NICF; these Rosen fractions are briefly described in the next section.

Although the appropriate terms are only defined in the following section, the reader may wish to compare Tong's Theorem with the following, whose proof appears in Section 3. (The constants $\tau_{k}$ are given in the statement of Theorem 10.)

Theorem 4. Fix an even $q=2 p$, with $p \geq 2$. For every $G_{q}$-irrational number $x$ and all positive $n$ and $k$, one has

$$
\min \left\{\Theta_{n-1}, \Theta_{n}, \ldots, \Theta_{n+k(p-1)}\right\}<\frac{-\tau_{k-1}}{1+(\lambda-1) \tau_{k-1}} .
$$

The constant $c_{k-1}=\frac{-\tau_{k-1}}{1+(\lambda-1) \tau_{k-1}}$ is best possible.

We prove an analogous result for all odd indices of these $G_{q}$ in Section 4 . In both cases, we prove a Borel-type result. Furthermore, our approach allows us to give various metric results.

\section{Rosen continued fractions}

In 1954, David Rosen (see [R]) introduced a family of continued fractions now bearing his name. The Rosen fractions form an infinite family of continued fractions generalizing the NICF. Although Rosen introduced his continued fractions to study certain Fuchsian groups, we are only concerned with their Diophantine approximation properties. 
Define $\lambda_{q}=2 \cos \frac{\pi}{q}$ for each $q \in\{3,4, \ldots\}$. For $q$ fixed, to simplify notation we usually write $\lambda$ for $\lambda_{q}$. For each $q$ the Rosen or $\lambda$-expansion $(\lambda \mathrm{CF})$ of $x$ is found by using the map $f_{q}:\left[-\frac{\lambda}{2}, \frac{\lambda}{2}\right) \rightarrow\left[-\frac{\lambda}{2}, \frac{\lambda}{2}\right)$, defined by

$$
f_{q}(x)=\frac{\varepsilon}{x}-\lambda r(x), x \neq 0 ; \quad f_{q}(0)=0,
$$

where $r(x)=\left\lfloor\frac{\varepsilon}{\lambda x}+\frac{1}{2}\right\rfloor$ and $\frac{\varepsilon}{x}=1 /|x|$. We usually write $r$ instead of $r(x)$. Since $\lambda_{3}=1$, we see that for $q=3$ the map $f_{q}$ is the NICF-operator $T_{\frac{1}{2}}$ from (1.2). For $x \in[-\lambda / 2, \lambda / 2)$, the map $f_{q}$ yields a continued fraction of the form

$$
x=\frac{\varepsilon_{1}}{r_{1} \lambda+\frac{\varepsilon_{2}}{r_{2} \lambda+\ldots+\frac{\varepsilon_{n}}{r_{n} \lambda+\ldots}}}=:\left[\varepsilon_{1}: r_{1}, \varepsilon_{2}: r_{2}, \ldots, \varepsilon_{n}: r_{n}, \ldots\right],
$$

where $\varepsilon_{i} \in\{ \pm 1\}$ and $r_{i} \in \mathbb{N}$. As usual, finite truncations yield the convergents $R_{n} / S_{n}$, for $n \geq 0$, i.e., $R_{n} / S_{n}=\left[\varepsilon_{1}: r_{1}, \varepsilon_{2}: r_{2}, \ldots, \varepsilon_{n}: r_{n}\right]$. The (Rosen) approximation coefficients of $x$ are defined by

$$
\Theta_{n}=\Theta_{n}(x)=S_{n}^{2}\left|x-\frac{R_{n}}{S_{n}}\right|, \quad \text { for } n \geq 0 .
$$

For $x \in[-\lambda / 2, \lambda / 2)$, we define the future $\left(t_{n}\right)$ and the past $\left(v_{n}\right)$ of $x$ at time $n$ by

$$
t_{n}=\left[\varepsilon_{n+1}: r_{n+1}, \varepsilon_{n+2}: r_{n+2}, \ldots\right], \quad v_{n}=\left[1: r_{n}, \varepsilon_{n}: r_{n-1}, \ldots, \varepsilon_{2}: r_{1}\right] .
$$

The map $f_{q}$ acts as a one-sided shift on the Rosen expansion of $x: f_{q}^{n}(x)=$ $t_{n}$. We define the natural extension operator to keep track of both $t_{n}$ and $v_{n}$.

Definition. For a fixed $q$ the natural extension map $\mathcal{T}$ is given by

$$
\mathcal{T}(x, y)=\left(f_{q}(x), \frac{1}{r \lambda+\varepsilon y}\right) .
$$

In [BKS] it was shown that for every $q \geq 3$ there exists a region $\Omega_{q} \subset \mathbb{R}^{2}$, for which $\mathcal{T}: \Omega_{q} \rightarrow \Omega_{q}$ is bijective almost everywhere (with respect to an invariant measure, see Equation (3.1), that is absolutely continuous with respect to Lebesgue measure). In Section 3 (for $q$ even) and Section 4 (for $q$ odd) we recall the exact form of $\Omega_{q}$. See also [N1], where $\Omega_{q}$ was obtained for $q$ even.

For $x=\left[\varepsilon_{1}: r_{1}, \varepsilon_{2}: r_{2}, \ldots\right]$ one has $\mathcal{T}^{n}(x, 0)=\left(t_{n}, v_{n}\right)$. The approximation coefficients of $x$ can be given in terms of $t_{n}$ and $v_{n}$ (see also [DK]) as

$$
\Theta_{n-1}=\frac{v_{n}}{1+t_{n} v_{n}}, \quad \Theta_{n}=\frac{\varepsilon_{n+1} t_{n}}{1+t_{n} v_{n}} .
$$


For simplicity, we say that a real number $r / s$ is a $G_{q}$-rational if it has finite Rosen expansion, all other real numbers are called $G_{q}$-irrationals. In [HS], Haas and Series derived a Hurwitz-type result using non-trivial hyperbolic geometric techniques. They showed that for every $G_{q}$-irrational $x$ there exist infinitely many $G_{q}$-rationals $r / s$, such that $\Theta(x, r / s) \leq \mathcal{H}_{q}$, where $\mathcal{H}_{q}$ is given by

$$
\mathcal{H}_{q}= \begin{cases}\frac{1}{2} & \text { if } q \text { is even } \\ \frac{1}{2 \sqrt{\left(1-\frac{\lambda}{2}\right)^{2}+1}} & \text { if } q \text { is odd }\end{cases}
$$

In this paper we derive a Borel-type result, by showing that for every $G_{q^{-}}$ irrational $x$ there are infinitely many $n \geq 1$ such that $\Theta_{n} \leq \mathcal{H}_{q}$. The even and odd case differ and we treat them separately. In both cases we focus on regions where $\min \left\{\Theta_{n-1}, \Theta_{n}, \ldots\right\}<\mathcal{H}_{q}$.

In fact, the Borel-type result we derive does not immediately imply the Hurwitz-type result of Haas and Series. Nakada [N2] showed that the Legendre constant $L_{q}$ is smaller than $\mathcal{H}_{q}$ (recall that for the RCF this Legendre constant is $1 / 2$, thus is larger than the Hurwitz constant $1 / \sqrt{5}$ ). Still, the Haas and Series results can be proved using continued fraction properties by means of a map which yields the Rosen-convergents and the so-called first medians; see [KNS].

\section{Tong's spectrum for even indices $q=2 p$}

In this section $q$ is even, we fix $q=2 p$. The region of the natural extension $\Omega_{q}$ is the smallest region where $\mathcal{T}$ is bijective. Usually we write $\Omega$ instead of $\Omega_{q}$. We have the following result from [BKS].

Theorem 5. ([BKS]) The domain $\Omega$ upon which $\mathcal{T}$ is bijective is given by

$$
\Omega=\bigcup_{j=1}^{p} J_{j} \times K_{j} .
$$

Here $J_{j}$ is defined as follows: Let $\phi_{j}=T^{j}\left(-\frac{\lambda}{2}\right)$, then $J_{j}=\left[\phi_{j-1}, \phi_{j}\right)$ for $j \in\{1,2, \ldots, p-1\}$ and $J_{p}=\left[0, \frac{\lambda}{2}\right)$. Further, $K_{j}=\left[0, L_{j}\right]$ for $j \in$ $\{1,2, \ldots, p-1\}$ and $K_{p}=[0, R]$, where $L_{j}$ and $R$ are derived from the relations

$$
\left\{\begin{array}{ll}
\left(\mathcal{R}_{0}\right): & R=\lambda-L_{p-1} \\
\left(\mathcal{R}_{1}\right): & L_{1}=1 /(\lambda+R) \\
\left(\mathcal{R}_{j}\right): & L_{j}=1 /\left(\lambda-L_{j-1}\right) \\
\left(\mathcal{R}_{p}\right): & R=1 /\left(\lambda-L_{p-1}\right) .
\end{array} \quad \text { for } j \in\{2, \cdots, p-1\}\right.
$$




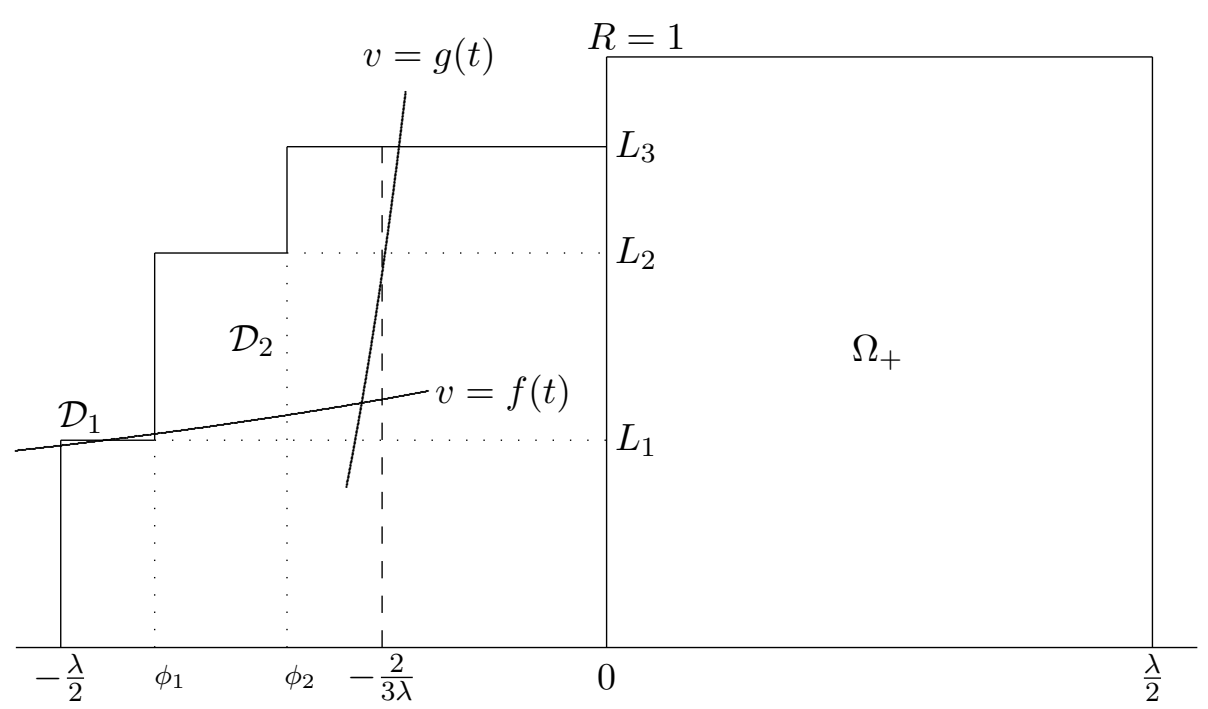

FiguRE 1. The region of the natural extension $\Omega_{8}$, with $\mathcal{D}$ of Lemma 7.

The map $f_{q}$ sends each interval $J_{i}$ to $J_{i+1}$ for $i=1, \ldots, p-1$. Further, we denote $\Omega_{+}=\{(t, v) \in \Omega \mid t>0\}$.

In $[\mathrm{BKS}]$ it is shown that $\mathcal{T}$ preserves a probability measure, $\nu$, that is absolutely continuous with respect to Lebesgue measure. Its density is

$$
g_{q}(t, v)= \begin{cases}\frac{C_{q}}{(1+t v)^{2}}, & \text { for }(t, v) \in \Omega_{q}, \\ 0, & \text { otherwise }\end{cases}
$$

where $C_{q}=\frac{1}{\log \left[\left(1+\cos \frac{\pi}{q}\right) / \sin \frac{\pi}{q}\right]}$ is a normalizing constant. It is also shown in $[\mathrm{BKS}]$, that the dynamical system $(\Omega, \nu, \mathcal{T})$ is weak Bernoulli (and therefore ergodic).

The following proposition on the distribution of the $\Theta_{n}$, also in [BKS], is a consequence of the Ergodic Theorem and the strong approximation properties of the Rosen fractions; see [DK], or [IK], Chapter 4.

Proposition 6. Let $q \geq 3$ be even. For almost all $G_{q}$-irrational numbers $x$ the two-dimensional sequence

$$
\mathcal{T}^{n}(x, 0)=\left(t_{n}, v_{n}\right), n \geq 1
$$

is distributed over $\Omega_{q}$ according to the density function $g_{q}(t, v)$ given in Equation (3.1). 
3.1. Consecutive pairs of large approximation constants: The region $\mathcal{D}$. To find Tong's spectrum we start by looking at two consecutive large approximation coefficients $\Theta_{n-1}$ and $\Theta_{n}$. In view of (2.2) we define $\mathcal{D} \subset \Omega$ by

$$
\mathcal{D}=\left\{(t, v) \in \Omega \mid \min \left\{\frac{v}{1+t v}, \frac{|t|}{1+t v}\right\}>\frac{1}{2}\right\} .
$$

So $\left(t_{n}, v_{n}\right) \in \mathcal{D}$ if and only if $\min \left\{\Theta_{n-1}, \Theta_{n}\right\}>\frac{1}{2}$. We have the following result describing $\mathcal{D}$.

Lemma 7. Define functions $f$ and $g$ by

$$
f(x)=\frac{1}{2-x} \quad \text { and } \quad g(x)=\frac{2|x|-1}{x} .
$$

For all even $q, \mathcal{D}$ consists of two connected components $\mathcal{D}_{1}$ and $\mathcal{D}_{2}$. The subregion $\mathcal{D}_{1}$ is bounded by the lines $t=-\frac{\lambda}{2}, v=L_{1}$ and the graph of $f ; \mathcal{D}_{2}$ is bounded by the graph of $g$ from the right, by the graph of $f$ from below and by the boundary of $\Omega$; see Figure 1 .

Proof. For the approximation coefficients one has

$$
\begin{aligned}
& \frac{v}{1+t v} \leq \frac{1}{2} \Leftrightarrow \quad v \leq f(t), \\
& \frac{|t|}{1+t v} \leq \frac{1}{2} \Leftrightarrow \begin{cases}v \leq g(t) & \text { if } t<0 \\
v \geq g(t) & \text { if } t \geq 0 .\end{cases}
\end{aligned}
$$

Since for $t>0$ the graphs of $f$ and $g$ meet at $t=1$, and $1>\frac{\lambda}{2}$, it follows that points for which $\min \left\{\frac{v}{1+t v}, \frac{|t|}{1+t v}\right\}<\frac{1}{2}$ must satisfy $t<0$ and $v>g(t)$. It is easy to check that for all even $q$ the only intersection points of the graphs of the functions $f$ and $g$ in the region of the natural extension are given by $\left(-\frac{\lambda}{2}, \frac{2}{\lambda+4}\right),\left(-L_{1}, L_{p-1}\right)$ and $\left(-L_{p-1}, L_{1}\right)$. The fact that $\mathcal{D}$ consists of the two pieces follows from $\phi_{0} \leq-L_{p-1}=1-\lambda \leq \phi_{1}$.

Having control on the approximation coefficients $\Theta_{n-1}$ and $\Theta_{n}$, we turn our attention to $\Theta_{n+1}$ on $\mathcal{D}$. It follows from (2.2) and the definition of $\mathcal{T}$ that

$$
\Theta_{n+1}=\frac{\varepsilon_{n+2}\left(1-\varepsilon_{n+1} r_{n+1} t_{n} \lambda\right)\left(\lambda r_{n+1}+\varepsilon_{n+1} v_{n}\right)}{1+t_{n} v_{n}}
$$

In order to express $\Theta_{n+1}$ locally as a function of only $t_{n}$ and $v_{n}$, we divide $\mathcal{D}$ into regions where $r_{n+1}, \varepsilon_{n+1}$ and $\varepsilon_{n+2}$ are constant. This gives three regions; see Table 1 for the definition of $\mathcal{A}, \mathcal{B}$, and $\mathcal{C}$, the new subregions involved in this. See also Figure 2. 


\begin{tabular}{ll|c|c|c|c} 
& Region & $r_{n+1}$ & $\varepsilon_{n+1}$ & $\varepsilon_{n+2}$ & $\Theta_{n+1}$ \\
$\mathcal{A}:$ & $\frac{-2}{3 \lambda} \leq t_{n} \leq \frac{-1}{\lambda+1}$ & 2 & -1 & -1 & $\frac{\left(1+2 t_{n} \lambda\right)\left(v_{n}-2 \lambda\right)}{1+t_{n} v_{n}}$ \\
$\mathcal{B}:$ & $\frac{-1}{\lambda} \leq t_{n}<\frac{-2}{3 \lambda}$ & 1 & -1 & 1 & $\frac{\left(1+t_{n} \lambda\right)\left(\lambda-v_{n}\right)}{1+t_{n} v_{n}}$ \\
$\mathcal{C} \cup \mathcal{D}_{1}:$ & $\frac{-\lambda}{2} \leq t_{n}<\frac{-1}{\lambda}$ & 1 & -1 & -1 & $\frac{\left(1+t_{n} \lambda\right)\left(v_{n}-\lambda\right)}{1+t_{n} v_{n}}$
\end{tabular}

TABLE 1. Subregions of $\mathcal{D}$ giving constant coefficients.

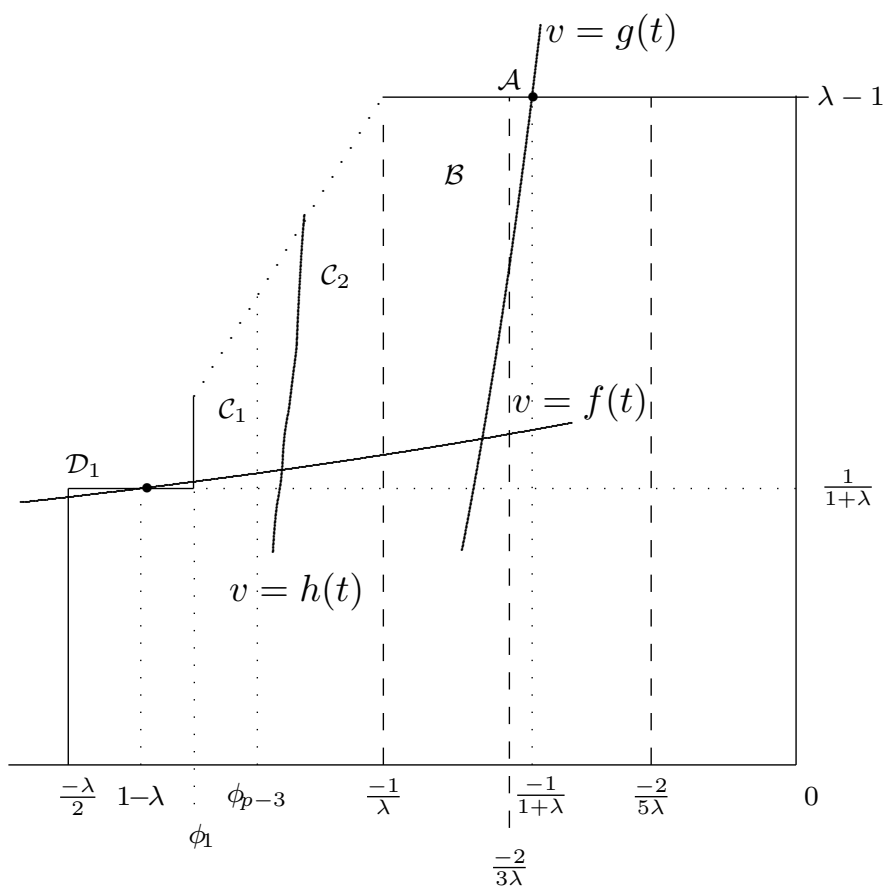

Figure 2. The regions in $\mathcal{D}$.

Solving for $\Theta_{n+1}=1 / 2$, leads to

$$
h(t)=\frac{2 \lambda^{2} t+2 \lambda+1}{2 \lambda t-t+2},
$$


whose graph divides $\mathcal{C}$ into two parts. Let $\mathcal{C}_{1}$ denote the left-hand side of this graph, there and on $\mathcal{D}_{1}$ one has $\Theta_{n+1}>\frac{1}{2}$; on the remainder, $\mathcal{C}_{2}$, one has $\Theta_{n+1}<\frac{1}{2}$. Note that $\mathcal{T}$ takes the graph of $h$ to the graph of $g$.

On its right-hand side region $\mathcal{B}$ is bounded by the graph of $g$. In view of Equation (3.4) and Table 1, we consider the graph of

$$
\ell(t)=\frac{2 \lambda^{2} t+2 \lambda-1}{2 \lambda t+t+2}, \quad \text { for } t \neq \frac{-2}{2 \lambda+1} .
$$

An easy calculation shows that the graphs of $\ell$ and $g$ intersect only at the point $(-1 /(\lambda+1), \lambda-1)$, and that for $t>-2 /(2 \lambda+1)$ the graph of $g$ lies above that of $\ell$. Furthermore, $\ell^{\prime}(t)>0$ for $t \neq \frac{-2}{2 \lambda+1}$, and $\ell\left(\frac{-1}{\lambda}\right)=\lambda$; we conclude that $\Theta_{n+1}<1 / 2$ on region $\mathcal{B}$.

Lemma 8. With notation as above, the subset of $\mathcal{D}$ on which $\Theta_{n+1}>1 / 2$ is exactly the union of regions $\mathcal{D}_{1}, \mathcal{C}_{1}$ and region $\mathcal{A}$. On region $\mathcal{A}$ one has $\Theta_{n+1}>\Theta_{n-1}>\Theta_{n}$.

Proof. The remarks directly above show that we need now only consider region $\mathcal{A}$.

It immediately follows from (2.2) and the fact that $v_{n}>-t_{n}$ on $\mathcal{A}$, that $\Theta_{n-1}>\Theta_{n}$. To show that $\Theta_{n+1}>\Theta_{n-1}$ we need to show

$$
\left(1+2 t_{n} \lambda\right)\left(v_{n}-2 \lambda\right)>v_{n} .
$$

or equivalently

$$
-4 \lambda^{2} t_{n}+2 \lambda t_{n} v_{n}-2 \lambda>0 .
$$

We use $v_{n}>-t_{n}$ again, so it is enough to show

$$
-4 \lambda^{2} t_{n}-2 \lambda t_{n}^{2}-2 \lambda \geq 0 \text {. }
$$

The last statement is true if $t_{n} \in\left[-\lambda-\sqrt{\lambda^{2}-1},-\lambda+\sqrt{\lambda^{2}-1}\right]$, which does indeed hold on region $\mathcal{A}$.

Since $\min \left\{\Theta_{n-1}, \Theta_{n}\right\}>\frac{1}{2}$ on $\mathcal{D}$, the result follows.

Now, by definition, $f_{q}(t)=-1 / t-\lambda$ for $t \in[-\lambda / 2,-2 / 3 \lambda)$. It follows that the $\mathcal{T}$ orbit of any point of the $\mathcal{C}_{i}$ either eventually leaves $\mathcal{D}$, or eventually enters $\mathcal{A}$. Thus, we naturally focus on the interval $t \in J_{p-1}=$ $\left[\phi_{p-2}, 0\right)$. For almost every $G_{q}$-irrational $x \in\left[-\frac{\lambda}{2}, \frac{\lambda}{2}\right)$ there is an $n$ such that $\mathcal{T}^{n}(x, 0)=\left(t_{n}, v_{n}\right)$ and $t_{n} \in J_{p-2}$. Divide the interval $J_{p-2}$ into three parts. If $t_{n} \in\left[\phi_{p-2}, \frac{-2}{3 \lambda}\right)$, then $\min \left\{\Theta_{n-1}, \Theta_{n}, \Theta_{n+1}\right\}<\frac{1}{2}$. If $t_{n} \in\left[\frac{-1}{\lambda+1}, 0\right)$, then $\left(t_{n}, v_{n}\right) \notin \mathcal{D}$ so $\min \left\{\Theta_{n-1}, \Theta_{n}\right\}<\frac{1}{2}$. However, if $t_{n} \in\left[\frac{-2}{3 \lambda}, \frac{-1}{\lambda+1}\right)$, then it may be that $\left(t_{n}, v_{n}\right) \in \mathcal{A}$ and thus $\min \left\{\Theta_{n-1}, \Theta_{n}, \Theta_{n+1}\right\}>\frac{1}{2}$.

Lemma 9. The transformation $\mathcal{T}$ maps $\mathcal{A}$ bijectively onto region $\mathcal{D}_{1}$. 
Proof. The vertices of $\mathcal{A}$ are mapped onto the vertices of region $\mathcal{D}_{1}$ by $\mathcal{T}$ :

$$
\begin{aligned}
\left(\frac{-2}{3 \lambda}, \lambda-1\right) & \mapsto\left(\frac{-\lambda}{2}, \frac{1}{\lambda+1}\right), \\
\left(\frac{-1}{\lambda+1}, \lambda-1\right) & \mapsto\left(1-\lambda, \frac{1}{\lambda+1}\right), \\
\left(\frac{-2}{3 \lambda}, \frac{3 \lambda-4}{2}\right) & \mapsto\left(\frac{-\lambda}{2}, \frac{2}{\lambda+4}\right) .
\end{aligned}
$$

It is easily checked that $\mathcal{T}$ takes the graph of $g$ to the graph of $f$. Since $\mathcal{T}$ is continuous and bijective and sends straight lines to straight lines, this completes the proof.

Under $\mathcal{T}$, region $\mathcal{D}_{1}$ is mapped onto a region with upper vertices $\left(\phi_{1}, L_{2}\right)$ and $\left(-L_{p-2}, L_{2}\right)$. For $i=2, \ldots, p-1$ we find a region with upper vertices $\left(\phi_{i-1}, L_{i}\right)$ and $\left(-L_{p-i}, L_{i}\right)$ after applying $\mathcal{T}^{i}$ to $\mathcal{A}$. The lower part of this region is bounded by the $i$ th transformation of the curve $g(t)$ under $\mathcal{T}$. After $p-1$ applications of $\mathcal{T}$ this results in a region with upper vertices $\left(\phi_{p-2}, L_{p-1}\right),\left(-L_{1}, L_{p-1}\right)$. Clearly, this region intersects with $\mathcal{A}$. We call $p-1$ consecutive applications of $\mathcal{T}$ a round.

That part of $\mathcal{T}^{p-1}(\mathcal{A})$ lying to the left of $t=\frac{-2}{3 \lambda}$ is in region $\mathcal{B}$; the images of these points under a subsequent application of $\mathcal{T}$ have positive $t$-coordinate. We call flushing an application of $\mathcal{T}$ to such points - the points are flushed from $\mathcal{D}$. The remainder of $\mathcal{T}^{p-1}(\mathcal{A})$ is a subset of $\mathcal{A}$.

Theorem 10. Any point $(t, v)$ of $\mathcal{A}$ is flushed after exactly $k$ rounds if and only if

$$
\tau_{k-1} \leq t<\tau_{k}
$$

where $\tau_{0}=\frac{-2}{3 \lambda}$ and

$$
\tau_{k}=\left[\left(-1: 2,(-1: 1)^{p-2}\right)^{k},\left(\frac{-2}{3 \lambda}:\right)\right] .
$$

For any $x$ with $\tau_{k-1} \leq t_{n}<\tau_{k}$,

$$
\min \left\{\Theta_{n-1}, \Theta_{n}, \ldots, \Theta_{n+k(p-1)-1}, \Theta_{n+k(p-1)}\right\}>\frac{1}{2},
$$

while

$$
\min \left\{\Theta_{n-1}, \Theta_{n}, \ldots, \Theta_{n+k(p-1)}, \Theta_{n+k(p-1)+1}\right\}<\frac{1}{2} .
$$

Proof. A point $(t, v) \in \mathcal{A}$ gets flushed after exactly $k$ rounds if $k$ is minimal such that $\mathcal{T}^{k(p-1)}(t, v)$ has its first coordinate smaller than $\frac{-2}{3 \lambda}$. We look at 
pre-images of $t=\frac{-2}{3 \lambda}$ under $f_{q}$ :

$$
\begin{aligned}
f_{q}^{-1}\left(\frac{-2}{3 \lambda}\right) & =\left[-1 ; 1,\left(\frac{-2}{3 \lambda}:\right)\right], \\
f_{q}^{-(p-1)}\left(\frac{-2}{3 \lambda}\right) & =\left[-1 ; 2,(-1: 1)^{p-2},\left(\frac{-2}{3 \lambda}:\right)\right], \\
& \vdots \\
f_{q}^{-k(p-1)}\left(\frac{-2}{3 \lambda}\right) & =\left[\left(-1 ; 2,(-1: 1)^{p-2}\right)^{k},\left(\frac{-2}{3 \lambda}:\right)\right]=\tau_{k} .
\end{aligned}
$$

The result thus clearly follows.

3.2. Metrical results. We define $\mathcal{A}_{k}=\left\{(t, v) \in \mathcal{A} \mid t \geq \tau_{k}\right\}$ for $k \geq 0$. From Theorem 10 and the ergodicity of $\mathcal{T}$ (cf. Proposition 6) we have the following metrical theorem on the distribution of large blocks of "big" approximation coefficients.

Theorem 11. For almost all $x$ (with respect to Lebesgue measure) and $k \geq 1$, the limit

$$
\begin{gathered}
\lim _{N \rightarrow \infty} \frac{1}{N} \#\left\{1 \leq j \leq N \mid \min \left\{\Theta_{j-1}, \Theta_{j}, \ldots, \Theta_{j+k(p-1)}\right\}>\frac{1}{2}\right. \\
\text { and } \left.\Theta_{j+k(p-1)+1}<\frac{1}{2}\right\}
\end{gathered}
$$

exists and equals $\nu\left(\mathcal{A}_{k-1} \backslash \mathcal{A}_{k}\right)=\nu\left(\mathcal{A}_{k-1}\right)-\nu\left(\mathcal{A}_{k}\right)$.

In order to apply Theorem 11 we compute $\nu(\mathcal{A})$ and $\nu\left(\mathcal{A}_{k}\right)$ :

$$
\begin{aligned}
\nu(\mathcal{A}) & =C_{q} \int_{t=\frac{-2}{3 \lambda}}^{-L_{1}} \int_{v=g(t)}^{L_{p-1}} \frac{1}{(1+t v)^{2}} d v d t \\
& =-C_{q} \int_{\frac{-2}{3 \lambda}}^{-L_{1}}\left(\frac{1}{2 t^{2}}+\frac{1}{t}-\frac{L_{p-1}}{1+L_{p-1} t}\right) d t \\
& =C_{q}\left(\frac{-1}{2 L_{1}}+\log \left|L_{p-1}+\frac{-1}{L_{1}}\right|+\frac{3 \lambda}{4}-\log \left|L_{p-1}-\frac{3 \lambda}{2}\right|\right) .
\end{aligned}
$$

Using the normalizing constant $C_{q}$ from Definition 3.1, $L_{1}=\frac{1}{\lambda+1}$ and $L_{p-1}=\lambda-1$, it follows from (3.5) that

$$
\nu(\mathcal{A})=\frac{1}{\log [(1+\lambda / 2) / \sin \pi / q]}\left(\frac{\lambda-2}{4}+\log \frac{4}{\lambda+2}\right) .
$$


Similarly,

$$
\begin{aligned}
\nu\left(\mathcal{A}_{k}\right) & =C_{q} \int_{t=\tau_{k}}^{-L_{1}} \int_{v=g(t)}^{L_{p-1}} \frac{1}{(1+t v)^{2}} d v d t \\
& =C_{q}\left[\frac{(-\lambda-1) \tau_{k}-1}{2 \tau_{k}}+\log \left|\frac{2 \tau_{k}}{(\lambda-1) \tau_{k}+1}\right|\right] .
\end{aligned}
$$

Example. If $q=8$ we have $\nu(\mathcal{A})=4.6 \cdot 10^{-4}, \nu\left(\mathcal{A}_{1}\right)=7.6 \cdot 10^{-7}$, and $\nu\left(\mathcal{A}_{2}\right)=6.7 \cdot 10^{-10}$. So Theorem 11 yields that for almost every $x$ about $4.6 \cdot 10^{-2} \%$ of the blocks of consecutive approximation coefficients of length 6 have the property that

$$
\min \left\{\Theta_{j-1}, \Theta_{j}, \ldots, \Theta_{j+3}\right\}>\frac{1}{2} \text { and } \Theta_{j+4}<\frac{1}{2},
$$

while about $7.6 \cdot 10^{-5} \%$ of the blocks of length 9 have the property that

$$
\min \left\{\Theta_{j-1}, \Theta_{j}, \ldots, \Theta_{j+6}\right\}>\frac{1}{2} \text { and } \Theta_{j+7}<\frac{1}{2}
$$

3.3. Tong's spectrum for even $\boldsymbol{q}$. We are now ready to determine the Tong spectrum for these Rosen continued fractions. Recall that Theorem 4 states that the minimum of $1+k(p-1)$ consecutive values of $\Theta_{j}$, beginning with $\Theta_{n-1}$ is less than $c_{k-1}:=\frac{-\tau_{k-1}}{1+(\lambda-1) \tau_{k-1}}$, where the $\tau_{j}$ are given in Theorem 10.

Proof of Theorem 4. We start with $k=1$. Assume that $\left(t_{n}, v_{n}\right) \in \mathcal{D}$, otherwise we are done. For a certain $0 \leq i \leq p-2$ we have $\left(t_{n+i}, v_{n+i}\right)$ is either in $\mathcal{A}$ or flushed to $\Omega_{+}$. In the latter case $\Theta_{n+i}<\frac{1}{2}$ and we are done. So assume that $(t, v)=\left(t_{n+i}, v_{n+i}\right) \in \mathcal{A}$. It follows from Lemma 8 that $\min \left\{\Theta_{n-1}, \Theta_{n}, \Theta_{n+1}\right\}=\Theta_{n}$ on $\mathcal{A}$. We give an upper bound for this minimum, by determining the maximum value of $\Theta_{n}$.

On $\mathcal{A}$ one has $\Theta_{n}=\frac{-t_{n}}{1+t_{n} v_{n}}$, so we must find the point $(t, v) \in \mathcal{A}$ where $m(t, v):=\frac{-t}{1+t v}$ attains its maximum. We have

$$
\frac{\partial m(t, v)}{\partial t}=\frac{-1}{(1+t v)^{2}}<0 \quad \text { and } \quad \frac{\partial m(t, v)}{\partial v}=\frac{t^{2}}{(1+t v)^{2}}>0
$$

Thus $\Theta_{n}$ attains its maximum at the upper left corner $\left(\frac{-2}{3 \lambda}, \lambda-1\right)$ of $\mathcal{A}$. This maximum equals $\frac{2}{\lambda+2}=c_{0}$.

It is left to show that $c_{0}$ is the best possible constant. We need to check that for the first $p-2$ points in either direction on the orbit of $\left(\frac{-2}{3 \lambda}, \lambda-1\right)$ 
one has $\min \left\{\Theta_{n-1}, \Theta_{n}\right\} \geq c_{0}$. These points on the orbit of $\left(\frac{-2}{3 \lambda}, \lambda-1\right)$ are

$$
\begin{aligned}
& \left(\left[(-1: 1)^{p-2},\left(\frac{-2}{3 \lambda}:\right)\right], L_{1}\right),\left(\left[(-1: 1)^{p-1},\left(\frac{-2}{3 \lambda}:\right)\right], L_{2}\right), \ldots, \\
& \left(\left[(-1: 1),\left(\frac{-2}{3 \lambda}:\right)\right], L_{p-2}\right),\left(\frac{-2}{3 \lambda}, \lambda-1\right),\left(\frac{-\lambda}{2}, L_{1}\right), \\
& \left(\phi_{1}, L_{2}\right),\left(\phi_{2}, L_{3}\right), \ldots,\left(\phi_{p-3}, L_{p-2}\right) .
\end{aligned}
$$

We consider the curves $\Theta_{n-1}=c_{0}$ and $\Theta_{n}=c_{0}$, thus

$$
f_{1}(x)=\frac{2}{\lambda+2-2 x} \quad \text { and } \quad g_{1}(x)=\frac{-(\lambda+2) x-2}{2 x} .
$$

The graph of $f_{1}(x)$ intersects the $x$-axis at the point $\left(0, \frac{2}{\lambda+2}\right)$. For the heights $L_{i}$ we easily find that $L_{p-2}>\ldots>L_{3}>L_{2}>\frac{2}{\lambda+2}$. Thus, each of the first $p-2$ points in either direction of the orbit has either $y$-coordinate greater than $L_{2}$ or is one of the points $\left(-\frac{\lambda}{2}, L_{1}\right)$ or $\left(\left[(-1: 1)^{p-2},\left(\frac{-2}{3 \lambda}:\right)\right], L_{1}\right)$. At the point $\left(-\frac{\lambda}{2}, L_{1}\right)$ the value of $\Theta_{n-1}$ is exactly $c_{1}=\frac{2}{\lambda+2}$. Further, we know that $\frac{\partial \Theta_{n-1}}{\partial t_{n}}>0$ on region $\mathcal{C}$, so

$$
\Theta_{n-1}\left(\left[(-1: 1)^{p-2},\left(\frac{-2}{3 \lambda}:\right)\right], L_{1}\right)>\Theta_{n-1}\left(-\frac{\lambda}{2}, L_{1}\right) .
$$

Together this means that for all these points one finds $\Theta_{n-1} \geq c_{0}$. The graph of $g_{1}(x)$ intersects with the $x$-axis in the point $\left(\frac{-2}{\lambda+2}, 0\right)$. We have

$$
-\frac{\lambda}{2}<\phi_{1}<\ldots<\phi_{p-1}<\left[-1: 1,\left(\frac{-2}{3 \lambda}:\right)\right]<\frac{-2}{\lambda+2},
$$

so for the relevant points on the orbit of $\left(\frac{-2}{3 \lambda}, \lambda-1\right)$ we have $\Theta_{n}>c_{0}$.

This proves the case $k=1$. For general $k$, assume that the starting point $\left(t_{n}, v_{n}\right) \in \mathcal{A}$ did not get flushed during the first $k-1$ rounds, otherwise we are done. Consider again a point $(t, v)=\left(t_{n+i}, v_{n+i}\right)$ in $\mathcal{A}$ with $0 \leq i \leq p-2$. From Theorem 10 this means that $t \geq \tau_{k-1}$. We find that the maximum of $\Theta_{n}$ occurs at the upper-left corner of the region where $(t, v)$ is located. This maximum is given by

$$
\Theta_{n}\left(\tau_{k-1}, \lambda-1\right)=\frac{-\tau_{k-1}}{1+(\lambda-1) \tau_{k-1}}=c_{k-1}
$$

The proof that this is the best possible is similar to the case $k=1$.

Note that

$$
\lim _{k \rightarrow \infty} \tau_{k}=\left[\overline{\left(-1: 2,(-1: 1)^{p-2}\right)}\right]=\frac{-1}{\lambda+1}
$$


yielding

$$
\lim _{k \rightarrow \infty} \frac{-\tau_{k}}{1+(\lambda-1) \tau_{k}}=\frac{1}{2} .
$$

Lemma 12. Let $\mathcal{F}$ denote the fixed point set in $\mathcal{D}$ of $\mathcal{T}^{(p-1)}$. Then

(i) $\mathcal{F}=\left\{\mathcal{T}^{i}(-1 /(\lambda+1), \lambda-1) \mid i=0,1, \ldots, p-1\right\}$;

(ii) For every $x$ and every $n \geq 0,\left(t_{n}, v_{n}\right) \notin \mathcal{F}$;

(iii) For every $G_{q}$-irrational number $x$ there are infinitely many $n$ for which $\left(t_{n}, v_{n}\right) \notin \mathcal{D}$;

(iv) For each $i=0,1, \ldots, p-1$, let $x_{i}=f_{q}^{i}(-1 /(\lambda+1))$. Then for all $n \geq 0, \mathcal{T}^{n}\left(x_{i}, 0\right) \notin \mathcal{D}$. However, $\mathcal{T}^{k(p-1)}\left(x_{i}, 0\right)$ converges from below along the vertical line $x=x_{i}$ to $\mathcal{T}^{i}(-1 /(\lambda+1), \lambda-1)$.

Proof. (i) Since $\mathcal{T}^{(p-1)}$ fixes $(-1 /(\lambda+1), \lambda-1), \mathcal{F}$ certainly contains the $\mathcal{T}$-orbit of $(-1 /(\lambda+1), \lambda-1)$. On the other hand, any point of $\mathcal{D}$ not in this orbit is eventually flushed from $\mathcal{D}$.

(ii) The second coordinate of any element of $\mathcal{F}$ is $G_{q}$-irrational, but for all $x$, the points $\left(t_{n}, v_{n}\right)$ have $G_{q}$-rational second coordinate.

(iii) From the previous item, for any $G_{q}$-irrational $x$ the $\mathcal{T}$-orbit of $(x, 0)$ avoids $\mathcal{F}$, hence each time this orbit encounters $\mathcal{D}$, it is flushed out within a finite number of iterations. We conclude indeed that for every $G_{q}$-irrational $x$ there are infinitely many $n$ for which $\left(t_{n}, v_{n}\right) \notin \mathcal{D}$.

(iv) The final item is easily checked.

Combining Theorem 4, Equation (3.6) and the previous lemma, we have the following result.

Theorem 13. For every $G_{q}$-irrational $x$ there are infinitely many $n \in \mathbb{N}$ for which

$$
\Theta_{n} \leq \mathcal{H}_{q}=\frac{1}{2}
$$

The constant $1 / 2$ is best possible.

\section{Tong's spectrum for odd indices $q=2 h+3$}

The classical case of $q=3$ is Tong's result itself; a geometric argument is given in $[\mathrm{HK}]$. In our treatment, the case of $q=5$ is also exceptional and we do not give full details for it. See in particular the remarks after Lemmata 15 and 16.

The results for the odd case are derived similarly to those of the even case. However, this case has more complicated dynamics, complicating the arguments. We begin with the definition of the natural extension. 


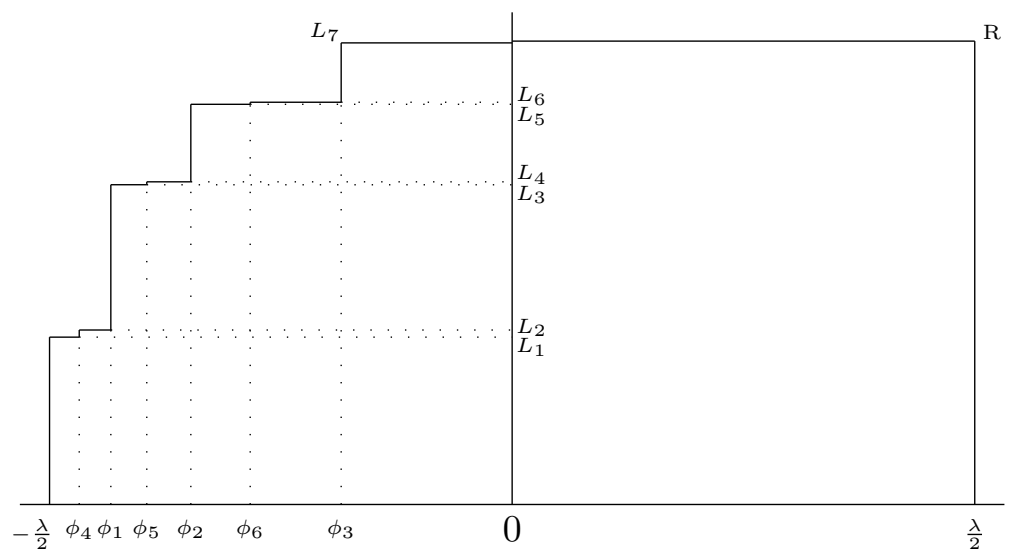

Figure 3. The region of the natural extension $\Omega_{9}$.

Again let $\phi_{j}=f_{q}^{j}(-\lambda / 2)$. Set $h=\frac{q-3}{2}$ and define $J_{j}, j \in\{1, \ldots, 2 h+2\}$, by

$$
\begin{aligned}
J_{2 k} & =\left[\phi_{h+k}, \phi_{k}\right), \text { for } k \in\{1, \ldots, h\}, \\
J_{2 k+1} & =\left[\phi_{k}, \phi_{h+k+1}\right), \text { for } k \in\{0,1, \ldots, h\},
\end{aligned}
$$

and $J_{2 h+2}=\left[0, \frac{\lambda}{2}\right)$. Let $K_{j}=\left[0, L_{j}\right]$ for $j \in\{1, \ldots, 2 h+1\}$ and let $K_{2 h+2}=$ $[0, R]$, where $R$ is the solution of

$$
R^{2}+(2-\lambda) R-1=0
$$

Theorem 14. ([BKS]) Let $q=2 h+3$, with $h \geq 1$ and $\Omega=\bigcup_{j=1}^{2 h+2} J_{j} \times K_{j}$. With $R$ defined as above, we have

$$
\left\{\begin{array}{ll}
\left(\mathcal{R}_{0}\right): & R=\lambda-L_{2 h+1}, \\
\left(\mathcal{R}_{1}\right): & L_{1}=1 /\left(2 \lambda-L_{2 h}\right), \\
\left(\mathcal{R}_{2}\right): & L_{2}=1 /\left(2 \lambda-L_{2 h+1}\right), \\
\left(\mathcal{R}_{j}\right): & L_{j}=1 /\left(\lambda-L_{j-2}\right) \\
\left(\mathcal{R}_{2 h+2}\right): & R=1 /\left(\lambda-L_{2 h}\right),
\end{array} \text { for } 2<j<2 h+2,\right.
$$

while $\mathcal{T}: \Omega \rightarrow \Omega$ is bijective off a set of Lebesgue measure zero.

In $[\mathrm{BKS}]$ it is shown that $\mathcal{T}$ preserves the probability measure $\nu$, with density $\frac{C_{q}}{(1+x y)^{2}}$, where $C_{q}=\frac{1}{\log (1+R)}$ is a normalizing constant. It is also shown in $[\mathrm{BKS}]$ that the process $(\Omega, \nu, \mathcal{T})$ is weak Bernoulli and therefore ergodic. Proposition 6 also holds in the odd case.

For odd indices $q$ we define again the region $\mathcal{D}$ by

$$
\mathcal{D}=\left\{(t, v) \in \Omega \mid \min \left\{\frac{v}{1+t v}, \frac{|t|}{1+t v}\right\}>\mathcal{H}_{q}\right\} .
$$




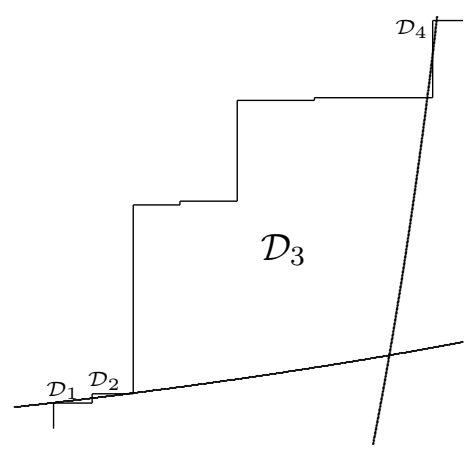

Figure 4. The region $\mathcal{D}$ for $q=9$.

Clearly $\min \left\{\Theta_{n-1}, \Theta_{n}\right\}>\mathcal{H}_{q}$ if and only if $\left(t_{n}, v_{n}\right) \in \mathcal{D}$.

Lemma 15. Define functions $f$ and $g$ by

$$
f(x)=\frac{\mathcal{H}_{q}}{1-\mathcal{H}_{q} x} \quad \text { and } g(x)=\frac{|x|-\mathcal{H}_{q}}{\mathcal{H}_{q} x} .
$$

For all odd $q \geq 7$, the region $\mathcal{D}$ consists of four disjoint regions:

$\mathcal{D}_{1}$ bounded by the lines $x=\frac{-\lambda}{2}$ and $y=L_{1}$ and the graph of $f$;

$\mathcal{D}_{2}$ bounded by the line $x=\phi_{h+1}$ and $y=L_{2}$ and the graph of $f$;

$\mathcal{D}_{3}$ (the largest region) bounded from below by the graph of $f$, from the right by the graph of $g$ and by the boundary of $\Omega$; and,

$\mathcal{D}_{4}$ bounded by the lines $x=\phi_{h}$ and $y=L_{2 h+1}$ and the graph of $g$.

Remark. In the exceptional case where $q=5$, one finds that $\mathcal{D}$ consists of a single connected component.

We begin our study of $\Theta_{n+1}$ by decomposing $\mathcal{D}$ into regions where $r_{n+1}, \varepsilon_{n+1}$ and $\varepsilon_{n+2}$ are constant; see Table 2 for the definition of the new subregions involved in this. See also Figure 5. Again, due to the dynamics of the situation, we will need only focus on the $\mathcal{T}$-orbit of $\mathcal{A}$.

On $\mathcal{A} \cup \mathcal{D}_{4}$, one easily finds that $\Theta_{n+1}>\Theta_{n-1}>\Theta_{n}$ holds. Again flushing occurs to the left of the line $t=\frac{-2}{3 \lambda}$; note that $\phi_{2 h} \leq \frac{-2}{3 \lambda}<\phi_{h}$. Thus we study the interval $\left[\phi_{2 h}, \phi_{h}\right)$ (instead of the interval $\left[\phi_{h}, 0\right)$, as in the even case). Note that the only one of our regions in the strip defined by the interval $\phi_{2 h} \leq t \leq \phi_{h}$ is indeed $\mathcal{A}$.

One easily shows the following result.

Lemma 16. The transformation $\mathcal{T}$ maps $\mathcal{A}$ bijectively to region $\mathcal{D}_{1}$.

Remark. In the exceptional case where $q=5$, one can easily see that $\mathcal{A}$ can be defined as in general, and has a similar image. The rest of our arguments can be appropriately adjusted so that the results announced below go through for $q=5$. 


\begin{tabular}{lc|c|c|c|c} 
& \multicolumn{1}{c|}{ Region } & $r_{n+1}$ & $\varepsilon_{n+1}$ & $\varepsilon_{n+2}$ & $\Theta_{n+1}$ \\
\hline $\mathcal{A} \cup \mathcal{D}_{4}:$ & $\frac{-2}{3 \lambda} \leq t_{n} \leq \frac{-1}{\lambda+\frac{1}{R}}$ & 2 & -1 & -1 & $\frac{\left(1+2 t_{n} \lambda\right)\left(v_{n}-2 \lambda\right)}{1+t_{n} v_{n}}$ \\
$\mathcal{B}:$ & $\frac{-1}{\lambda} \leq t_{n}<\frac{-2}{3 \lambda}$ & 1 & -1 & 1 & $\frac{\left(1+t_{n} \lambda\right)\left(\lambda-v_{n}\right)}{1+t_{n} v_{n}}$ \\
$\mathcal{C} \cup \mathcal{D}_{1} \cup \mathcal{D}_{2}:$ & $\frac{-\lambda}{2} \leq t_{n}<\frac{-1}{\lambda}$ & 1 & -1 & -1 & $\frac{\left(1+t_{n} \lambda\right)\left(v_{n}-\lambda\right)}{1+t_{n} v_{n}}$
\end{tabular}

TABLE 2. Regions of constant coefficients, the odd case.

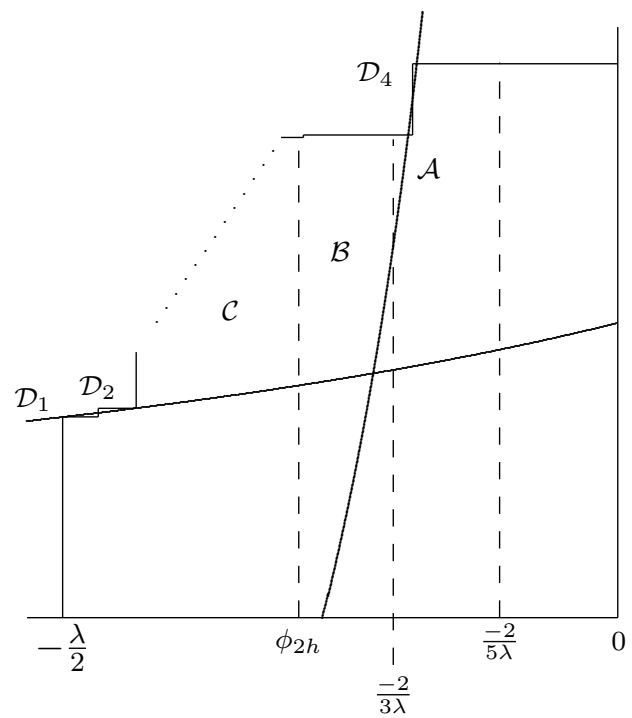

Figure 5. The regions for $\Theta_{n+1}$ on $\mathcal{D}$.

The transformations of $\mathcal{A}$ follow a more complex orbit than in the even case: here, the regions make a "double loop," related to the orbit of the $\phi_{j}$. Region $\mathcal{D}_{1}$ gets transformed into a region with upper right vertex $\left(-L_{2 h-1}, L_{3}\right)$, this region gets transformed in a region with upper right vertex $\left(-L_{2 h-3}, L_{5}\right)$ and so on until we reach a region with upper right corner $\left(-L_{1}, L_{2 h+1}\right)$, which lies in region $\mathcal{D}_{4}$. This region gets transformed into a region with upper right vertex $\left(-L_{2 h}, L_{2}\right)$. Thereafter we find a region with upper right corner $\left(-L_{2 h-2}, L_{4}\right)$ and so on, until finally the image intersects with $\mathcal{A}$ after $2 h+1$ steps. Here, we call a round these $2 h+1$ transformations of $\mathcal{T}$. 
Theorem 17. Let the constants $\tau_{k}$ be defined by

$$
\tau_{k}=\left[\left(-1: 2,(-1: 1)^{h},-1: 2,(-1: 1)^{h-1}\right)^{k},\left(\frac{-2}{3 \lambda}:\right)\right] .
$$

Then for any $(t, v) \in \mathcal{A}$ such that $\tau_{k-1} \leq t<\tau_{k}$, the point $\left(t_{n}, v_{n}\right)$ is flushed after $k$ rounds. In particular, for any $x \in \mathbb{R}$ with $\mathcal{T}^{n}(x, 0)=\left(t_{n}, v_{n}\right)$ satisfying $\tau_{k-1} \leq t_{n}<\tau_{k}$ one has

$$
\min \left\{\Theta_{n-1}, \Theta_{n}, \ldots, \Theta_{n+k(2 h+1)}, \Theta_{n+k(2 h+1)}\right\}>\mathcal{H}_{q},
$$

while

$$
\min \left\{\Theta_{n-1}, \Theta_{n}, \ldots, \Theta_{n+k(2 h+1)}, \Theta_{n+k(2 h+1)+1}\right\}<\mathcal{H}_{q}
$$

4.1. Metric results. As in the even case, we define

$$
\mathcal{A}_{k}=\left\{(t, v) \in \mathcal{A} \mid t>\tau_{k}\right\} \text { for } k \geq 0 .
$$

Theorem 18. For almost all $x$ (with respect to the Lebesgue measure) and $k \geq 1$ the limit

$$
\begin{gathered}
\lim _{N \rightarrow \infty} \frac{1}{N} \#\left\{1 \leq j \leq N \mid \min \left\{\Theta_{j-1}, \Theta_{j}, \ldots, \Theta_{j+k(2 h+1)}\right\}>\frac{1}{2}\right. \\
\text { and } \left.\Theta_{j+k(2 h+1)+1}<\frac{1}{2}\right\}
\end{gathered}
$$

exists and equals $\nu\left(\mathcal{A}_{k-1} \backslash \mathcal{A}_{k}\right)=\nu\left(\mathcal{A}_{k-1}\right)-\nu\left(\mathcal{A}_{k}\right)$.

We compute again $\nu(\mathcal{A})$ and $\nu\left(\mathcal{A}_{k}\right)$. A calculation similar to that in the even case yields

$$
\begin{aligned}
\nu(\mathcal{A}) & =D\left(\log \left(R+\frac{1}{R}\right)-\log \left(\frac{\lambda R+2}{2 R}\right)+\frac{\lambda C-2 C R}{2}\right), \\
\nu\left(\mathcal{A}_{k}\right) & =D\left(\log \left(R+\frac{1}{R}\right)-\log \left|\frac{1+\tau_{k}(\lambda-1 / R)}{\tau_{k}}\right|-C(\lambda+R)-\frac{C}{\tau_{k}}\right) .
\end{aligned}
$$

Example. If $q=9$ we have $\nu(\mathcal{A})=6.2 \cdot 10^{-7}, \nu\left(\mathcal{A}_{1}\right)=6.5 \cdot 10^{-13}$, and $\nu\left(\mathcal{A}_{2}\right)=6.8 \cdot 10^{-19}$.

So we find that for almost every $x$ about $6.5 \cdot 10^{-11} \%$ of the blocks of consecutive approximation coefficients of length 10 have the property that

$$
\min \left\{\Theta_{j-1}, \Theta_{j}, \ldots, \Theta_{j+7}\right\}>\frac{1}{2} \text { and } \Theta_{j+8}<\frac{1}{2},
$$

while about $6.8 \cdot 10^{-17} \%$ of the blocks of length 17 have the property that

$$
\min \left\{\Theta_{j-1}, \Theta_{j}, \ldots, \Theta_{j+14}\right\}>\frac{1}{2} \text { and } \Theta_{j+15}<\frac{1}{2} .
$$


4.2. Tong's spectrum for odd $\boldsymbol{q}$. We have the following result, which is proved similarly to Theorem 4 .

Theorem 19. For every $G_{q}$-irrational number $x$ and all positive $n$ and $k$, one has

$$
\min \left\{\Theta_{n-1}, \Theta_{n}, \ldots, \Theta_{n+k(2 h+1)}\right\}<\frac{-\tau_{k-1}}{1+(\lambda-R) \tau_{k-1}}
$$

The constant $c_{k-1}=\frac{-\tau_{k-1}}{1+(\lambda-R) \tau_{k-1}}$ is best possible.

Note that $\lim _{k \rightarrow \infty} c_{k}=\mathcal{H}_{q}$. Due to this, and reasoning as in the proof of Lemma 12 (here one considers the fixed-points in $\mathcal{D}$ of $\mathcal{T}^{2 h+1}$ instead of those of $\mathcal{T}^{p-1}$ ), we get the following result.

Theorem 20. For every $G_{q}$-irrational $x$ there are infinitely many $n \in \mathbb{N}$, such that

$$
\Theta_{n}(x) \leq \mathcal{H}_{q}
$$

The constant $\mathcal{H}_{q}$ is best possible.

Acknowledgements. We thank the referee for a careful reading of this paper.

\section{References}

[A] Adams, William W., On a relationship between the convergents of the nearest integer and regular continued fractions. Math. Comp. 33 (1979), no. 148, 1321-1331.

[B] Burger, E.B., Exploring the number jungle: a journey into Diophantine analysis. Student Mathematical Library, 8. American Mathematical Society, Providence, RI, 2000.

[BJW] Bosma, W., Jager, H. And WiediJk, F., Some metrical observations on the approximation by continued fractions. Nederl. Akad. Wetensch. Indag. Math. 45 (1983), no. 3, 281-299.

[BKS] Burton, R.M., Kraaikamp, C. And Schmidt, T.A., Natural extensions for the Rosen fractions. Trans. Amer. Math. Soc. 352 (1999), 1277-1298.

[CF] Cusick, T.W. And Flahive, M.E., The Markoff and Lagrange spectra. Mathematical Surveys and Monographs, 30. American Mathematical Society, Providence, RI, 1989.

[DK] Dajani, K. and Kraaikamp, C., Ergodic Theory of Numbers. The Carus Mathematical Monographs 29 (2002).

[IK] Iosifescu, M. and KraAikamp, C., Metrical Theory of Continued Fractions. Mathematics and its Applications, 547. Kluwer Academic Publishers, Dordrecht, 2002.

[HK] Hartono, Y. and KraAikamp, C., Tong's spectrum for semi-regular continued fraction expansions. Far East J. Math. Sci. (FJMS) 13 (2004), no. 2, 137-165.

[HS] HAAs, A. AND SerIEs, C., Hurwitz constants and Diophantine approximation on Hecke groups. J. London Math. Soc. 34 (1986), 219-234.

[JK] Jager, H. And KraAikamp, C., On the approximation by continued fractions. Nederl. Akad. Wetensch. Indag. Math. 51 (1989), no. 3, 289-307.

[KNS] KraAikamp, C., NAKAdA, H. AND Schmidt, T.A., On approximation by Rosen continued fractions. In preparation (2006).

[L] Legendre, A. M., Essai sur la théorie des nombres. Paris (1798).

[N1] NAKAdA, H., Continued fractions, geodesic flows and Ford circles. Algorithms, Fractals and Dynamics, (1995), 179-191.

[N2] NAKADA, H., On the Lenstra constant. Submitted (2007), eprint: arXiv: 0705.3756. 
[R] Rosen, D., A class of continued fractions associated with certain properly discontinuous groups. Duke Math. J. 21 (1954), 549-563.

[T1] Tong, J.C., Approximation by nearest integer continued fractions. Math. Scand. 71 (1992), no. 2, 161-166.

[T2] Tong, J.C., Approximation by nearest integer continued fractions. II. Math. Scand. 74 (1994), no. 1, 17-18.

Cornelis KraAiKAmP

EWI, Delft University of Technology,

Mekelweg 4, 2628 CD Delft

the Netherlands

E-mail: c.kraaikamp@tudelft.nl

Thomas A. SchmidT

Oregon State University

Corvallis, OR 97331

USA

E-mail: toms@math.orst.edu

Ionica SMEETS

Mathematical Institute

Leiden University

Niels Bohrweg 1, 2333 CA Leiden

the Netherlands

E-mail: smeets@math.leidenuniv.nl 